\title{
2DPackLib: a two-dimensional cutting and packing library
}

\section{Manuel lori ${ }^{1} \cdot$ Vinícius Loti de Lima $^{2} \cdot$ Silvano Martello $^{3} \cdot$ Michele Monaci $^{3}$}

Received: 17 April 2021 / Accepted: 23 September 2021 / Published online: 12 October 2021

(c) The Author(s) 2021

\begin{abstract}
Two-dimensional cutting and packing problems model a large number of relevant industrial applications. The literature on practical algorithms for such problems is very large. We introduce the 2DPackLib, a library on two-dimensional orthogonal cutting and packing problems. The library makes available, in a unified format, 25 benchmarks from the literature for a total of over 3000 instances, provides direct links to surveys and typologies, and includes a list of relevant links.
\end{abstract}

Keywords Two-dimensional cutting and packing $\cdot$ Library $\cdot$ Benchmarks

\section{Introduction}

Cutting and packing is a major field in optimization. Two-dimensional (rectangular) cutting and packing problems model indeed a large number of relevant industrial applications. In the former class of problems a set of rectangular items must be cut from a set of rectangular stock units, whereas in the latter a set of rectangular items must be packed into a set of rectangular containers. It is thus clear that the two problems are strongly related, in the sense that cutting an item from a stock unit is practically equivalent to packing an item into a container. In the following, we use the terms cutting and packing interchangeably and we refer to both stock units and containers as

$凶$ Silvano Martello

silvano.martello@unibo.it

Manuel Iori

manuel.iori@unimore.it

Vinícius Loti de Lima

v.loti@ic.unicamp.br

Michele Monaci

michele.monaci@unibo.it

1 DISMI, University of Modena and Reggio Emilia, Reggio Emilia, Italy

2 Institute of Computing, University of Campinas, Campinas, Brazil

3 DEI "Guglielmo Marconi”, University of Bologna, Bologna, Italy 
bins. Typical cutting applications derive from industrial productions involving steel, wood or glass, where the aim is to reduce waste material. Typical packing applications include optimal use of spaces in warehouses or in packaging processes. According to specific applications, two-dimensional cutting and packing problems may require to optimize different objective functions, and to satisfy specialized side constraints.

In the following, we deal with the most studied variants of these problems, where the edges of the items must be parallel to those of the bin in which they are packed (orthogonal rectangular cutting and packing). Let $\mathcal{B}=(W, H)$ be a rectangular bin of width $W \in \mathbb{Z}_{+}$and height $H \in \mathbb{Z}_{+}$, and $\mathcal{I}$ be a set of rectangular items, where each item $i \in \mathcal{I}$ has width $w_{i} \in \mathbb{Z}_{+}$and height $h_{i} \in \mathbb{Z}_{+}$(with $w_{i} \leq W$ and $h_{i} \leq H$ ). A packing of $\mathcal{I}^{\prime} \subseteq \mathcal{I}$ into $\mathcal{B}$ can be described as a function $\mathcal{F}: \mathcal{I}^{\prime} \rightarrow \mathbb{Z}_{+}^{2}$ that maps each item $i \in \mathcal{I}^{\prime}$ to a pair of coordinates $\mathcal{F}(i)=\left(x_{i}, y_{i}\right)$, such that

$$
\begin{aligned}
& x_{i} \in\left\{0, \ldots, W-w_{i}\right\} \text { and } y_{i} \in\left\{0, \ldots, H-h_{i}\right\}\left(i \in \mathcal{I}^{\prime}\right), \\
& {\left[x_{i}, x_{i}+w_{i}\right) \cap\left[x_{j}, x_{j}+w_{j}\right)=\emptyset \text { or }\left[y_{i}, y_{i}+h_{i}\right) \cap\left[y_{j}, y_{j}+h_{j}\right)} \\
& =\emptyset\left(i, j \in \mathcal{I}^{\prime}, i \neq j\right) .
\end{aligned}
$$

In this representation, the bin is located on a Cartesian plane with its edges parallel to the axes and its bottom-left corner on the origin. The coordinates $\mathcal{F}(i)=\left(x_{i}, y_{i}\right)$ represent the position where the bottom-left corner of each $i \in \mathcal{I}^{\prime}$ is placed. Constraints (1) impose that each item is entirely inside the bin, while constraints (2) forbid overlapping between pairs of items. Constraints (1) and (2) impose that items have fixed orientation (i.e., cannot be rotated), but they can be adapted to consider the popular variant (discussed in Sect. 2) where items can be rotated by 90 degrees.

Iori et al. [26] recently presented a survey on the main exact solution methods for the most popular two-dimensional orthogonal cutting and packing problems. The huge number of algorithms proposed for these problems has involved, along the years, the adoption of many benchmarks for evaluating their performance. The survey provides a precise classification of the different problem variants. Concerning benchmarks and computational experiments, some issues must be considered:

- The number of benchmarks in the literature is huge, and even instances proposed in the Seventies are still used to evaluate new methods;

- There is no clear correspondence between tested variants and adopted benchmarks as, due to similarity among some of the variants, the same benchmarks have been adopted for different variants;

- Many benchmarks are available in different specific libraries, where they are provided in different formats, not always clearly detailed;

- Some benchmarks were originally published in personal web pages which no longer exist.

Besides the classification of variants and benchmarks, the authors implemented a standardization of the benchmarks and a unified format for the instances, as well as an analysis of the matching variants-benchmarks. We now collected these results and other useful instruments (visual tools, links to surveys, bibliographies) in a publicly available web page, the $2 \mathrm{DPackLi}$. 
Web-based libraries for combinatorial optimization problems have become a popular instrument for researchers wanting to study specific fields and to test their findings on reliable benchmarks. The seminal instruments of this kind, realized 30 years ago, are still very active: the OR-Library, developed in 1990 by Beasley [6] (see http:// people.brunel.ac.uk/ mastjjb/jeb/info.html), mostly devoted to a number of combinatorial optimization problems, and the QAPLIB, implemented in 1991 by Burkard et al. [11,12] for the Quadratic Assignment Problem (see https://coral.ise.lehigh.edu/ data-sets/qaplib/). In the following decade, Applegate et al. [3] presented a famous, rich web page (see http://www.math.uwaterloo.ca/tsp/) for the Traveling Salesman Problem. In the last ten years, new libraries have been implemented for Mixed Integer Programming (the MIPLIB by Koch et al. [27], see http://miplib.zib.de/), for Vehicle Routing (the VRPH by Groër et al. [22], see http://sites.google.com/site/vrphlibrary/, and the CVRPLIB by Uchoa et al. [45], see http://vrp.atd-lab.inf.puc-rio.br/), and for Bin Packing (the BPPLIB by Delorme et al. [18], see http://or.dei.unibo.it/library/ bpplib).

The purpose of this paper is to present the contents of the 2DPackLib and to provide systematized relationships and links that should hopefully assist researchers in the development and testing of new solution approaches.

The library is available at http://or.dei.unibo.it/library/2dpacklib. In the next sections, we describe its contents, namely:

- Synthetic classification of problems and variants;

- Pointers to surveys and typologies;

- Standardized benchmarks and their matching with problems and variants;

- Bibliographies and additional tools.

\section{Classification}

Using the basic definitions provided in Sect. 1, the 2DPackLib classifies the main two-dimensional orthogonal cutting and packing problems as follows:

- Two-Dimensional Strip Packing Problem (2D-SPP): given a set $\mathcal{I}$ of items and a width $W$, determine the minimum $H$ value such that there exists a packing of $\mathcal{I}$ into a bin $\mathcal{B}=(W, H)$. In this problem, there is a unique container, called strip, having a fixed width and an infinite height;

- Two-Dimensional Cutting Stock Problem (2D-CSP): given an unlimited number of identical bins and a set $\mathcal{I}$ of items, where each item is associated with a demand $d_{i} \in \mathbb{Z}_{+}$(the minimum number of copies to be packed), determine the minimum number of bins needed to pack all demands. The Two-Dimensional Bin Packing Problem (2D-BPP) is the special case in which $d_{i}=1$, for all $i \in \mathcal{I}$;

- Two-Dimensional Knapsack Problem (2D-KP): given a bin $\mathcal{B}$ and a set $\mathcal{I}$ of items, where each item $i \in \mathcal{I}$ is associated with a profit $p_{i}$, determine a subset $\mathcal{I}^{\prime} \subseteq \mathcal{I}$ that can be packed into $\mathcal{B}$ and such that $\sum_{i \in I^{\prime}} p_{i}$ is a maximum. In the $2 \mathrm{D}-\mathrm{KP}$, each item $i \in \mathcal{I}$ may be associated with a maximum number of copies $b_{i}$. This version of the problem is known as the constrained $2 D-K P$, whereas in the unconstrained version of the problem an unlimited number of copies of each item is available; 
Table 1 Problem classifications according to classical typologies

\begin{tabular}{llll}
\hline & Dyckhoff [19] & Lodi et al. [31] & Wäscher et al. [48] \\
\hline $2 \mathrm{D}-\mathrm{SPP}$ & - & $2 \mathrm{SP}|\mathrm{O}| \mathrm{F}$ & ODP \\
$2 \mathrm{D}-\mathrm{BPP}$ & $2 / \mathrm{V} / \mathrm{I} / \mathrm{M}$ & $2 \mathrm{BP}|\mathrm{O}| \mathrm{F}$ & SBSBPP \\
$2 \mathrm{D}-\mathrm{CSP}$ & $2 / \mathrm{V} / \mathrm{R}$ & $2 \mathrm{BP}|\mathrm{O}| \mathrm{F}$ & SSSCSP \\
$2 \mathrm{D}-\mathrm{KP}$ & $2 / \mathrm{B} / \mathrm{O}$ & $2 \mathrm{KP}|\mathrm{O}| \mathrm{F}$ & SLOPP \\
\hline
\end{tabular}

- Two-Dimensional Orthogonal Packing Problem (2D-OPP): given a bin $\mathcal{B}$ and a set $\mathcal{I}$ of items, determine whether there exists a packing of $\mathcal{I}$ into $\mathcal{B}$.

Many variants of these problems have been studied in the literature, the most popular ones being:

- guillotine cuts: in each bin, cuts are restricted to be edge-to-edge and parallel to the edges of the bin. A cut splits a bin into two rectangles, which in turn can be waste, items, or bins to be recursively cut again;

- orthogonal rotations: items are allowed to be rotated by 90 degrees, as opposed to having fixed orientation;

- variable-sized bins: this variant arises when, instead of an unlimited number of identical bins, one is given a set of bin types, each having a specific size (width and height), cost, and availability. Typically, this variant is studied for problems 2D-CSP and 2D-BPP;

- loading and unloading constraints: a packing is associated with a loading/unloading item sequence and must be such that each item can be loaded/unloaded with no need to physically repositioning other items.

These problems are strongly $\mathcal{N} \mathcal{P}$-hard (the $2 \mathrm{D}$-OPP, strongly $\mathcal{N} \mathcal{P}$-complete) and very difficult to solve in practice. The combinations problem-variant produce a high number of specific issues, and many of them have been investigated in the literature. (The survey [26] examines over 180 related references.)

\section{Surveys and typologies}

In 1990, Dyckhoff [19] proposed the first typology for classifying cutting and packing problems. Other typologies were later developed by Lodi et al. [31] (further extended by Martello et al. [34]) and Wäscher et al. [48]. Table 1 provides the classification of the optimization problems considered in the 2DPackLib according to the different typologies. The classification by Lodi et al. [31] also covers the variants that consider orthogonal rotations (' $\mathrm{R}$ ' instead of ' $\mathrm{O}$ ' in the second field) and guillotine cuts (' $\mathrm{G}$ ' instead of ' $F$ ' in the third field).

In the Noughties, surveys on two-dimensional packing problems were presented by Lodi et al. [29,32], while Ntene and van Vuuren [38] produced a specialized review and comparison of guillotine heuristics for the 2D-SPP.

In the following decade, a survey on general two-dimensional packing problems was proposed by Alvarez-Valdes et al. [1]. Surveys on specific problems, variants, 
and methodologies were produced by Lodi et al. [30] (2D-BPP and 2D-SPP), Silva et al. [44] (pallet loading problem, a variant of the 2D-BPP in which one has to pack, with orthogonal rotations, the maximum number of identical items into a single bin), Oliveira et al. [40] (2D-SPP), Christensen et al. [14] (approximation and online algorithms), and Russo et al. [41] (upper bounds for problems with guillotine cuts).

Finally, in the last two years, Bezerra et al. [10] proposed a survey on a special case of the 2D-SPP, while Iori et al. [26] reviewed exact algorithms for two-dimensional cutting and packing problems.

The 2DPackLib provides direct pointers to the PDF files of all surveys mentioned above (journal subscriptions may be requested).

\section{Benchmarks}

This is the most relevant section of the 2DPackLib. Many benchmarks have been proposed to evaluate the computational performances of algorithms for different variants of two-dimensional cutting and packing problems. Unfortunately the literature has a number of drawbacks: unclear correspondence between variants and benchmarks, non-uniform data formats, sometimes hard to understand, unavailability of benchmarks included in dead personal web pages.

In order to facilitate future computational studies, we converted the main benchmark sets to a standard format that comprises the four main 2D cutting and packing problems with identical bins. For that, let $W$ and $H$ be, respectively, the width and height of the bin, and $w_{i}, h_{i}, d_{i}, b_{i}$, and $p_{i}$ the width, height, demand, maximum number of copies, and profit of each item $i \in \mathcal{I}=\{1, \ldots, m\}$. The instances are in the following format:

- $m$

- $W H$

- For each item $i(i=1, \ldots, m): i w_{i} h_{i} d_{i} b_{i} p_{i}$

The width and height of the items appear in all problems. The demands appear in the 2D-CSP and, sometimes, in variants of the 2D-SPP and the 2D-OPP. The maximum number of copies of items is usually considered in the constrained 2D-KP and variants. The profit is considered in the 2D-KP only.

The format can obviously be used for the variants that include guillotine cuts and orthogonal rotations as well, but not for the cases of loading/unloading constraints and variable-sized bins. However, very few benchmarks have been proposed for these two cases, and a more general format for handling them would be considerably more complex for the most commonly used benchmarks. The library provides however pointers to benchmarks for a number of variants and related problems that do not fall in our format.

The benchmark sets can be downloaded, in the unified format, from the 2DPackLib as Zip files. Since most benchmarks have been adopted for different problem variants, the library also provides, for each benchmark, an up-to-date list of recently published results on different problems. The next sections provide a synthetic description of the benchmark sets. 


\subsection{Benchmarks originally proposed for the 2D-SPP}

- $\mathbf{N}$ and T: two benchmarks, composed by 35 instances each, proposed by Hopper and Turton [25]. The number of items ranges between 17 and 199 and the strip width is always equal to 200 ;

- C: 21 instances proposed by Hopper and Turton [24], in which the number of items ranges between 16 and 197, and the width of the strip ranges between 20 and 160 . The instances are generated so that the optimal solution is known in advance. A subset of 9 instances from this benchmark has been referred to as "HT" by some authors;

- BKW: 13 instances proposed by Burke et al. [13], in which the number of items ranges between 10 and 3152, and the width of the strip ranges between 40 and 640 . These instances, that are also referred to as "n" by some authors, were generated so that an optimal solution consists of a perfect packing that can be produced by guillotine cuts;

- ZDF: 16 instances produced by Leung and Zhang [28] by combining other instances from the literature. The number of items ranges between 580 and 75032 , and the width of the strips ranges between 100 and 9000 .

The files provided for 2D-SPP instances also report a value for $\mathrm{H}$, which is either the optimal height or -1 . The first case only occurs for benchmarks where the optimal solution was already produced by the generation process.

\subsection{Benchmarks proposed for the 2D-BPP and 2D-CSP}

- BENG: ten instances introduced by Bengtsson [8] by generating both the size of the items and the dimensions of the bins according to a uniform distribution. The number of items ranges between 20 and 200, whereas the largest bin is $(40,25)$.

- CLASS: this benchmark includes 500 instances, divided into 10 classes, and was introduced by Berkey and Wang [9] (classes 1 to 6) and by Martello and Vigo [35] (classes 7 to 10). Each class includes instances of different sizes, namely it has 10 instances for each value of the number of items in $\{20,40,60,80,100\}$. The bins are identical for all instances in each class, and they vary from $(10,10)$ in class 1 to $(300,300)$ in class 6 ;

- A: 43 instances introduced by Macedo et al. [33] for the 2D-CSP with guillotine cuts. In these instances, that are derived from the furniture industry, the number of items ranges between 13 and 809 and the bins are either $(2750,1220)$, $(2550,2100)$, or $(2470,2080)$.

\subsection{Benchmarks proposed for the 2D-KP}

- CGCUT: three small instances generated by Christofides and Whitlock [15]. The dimensions and profits of the items were generated following a uniform distribution. Some authors refer to this set as "ChW"; 
- WANG: three small instances obtained by Wang [47] by modifying the bin and the maximum number of copies of one instance from CGCUT, and by considering the profit of each item equal to its area;

- NGCUT: 12 small instances generated by Beasley [5]. The dimensions and profits of the items were generated following a uniform distribution;

- GCUT: 13 instances proposed by Beasley [4]. The first 12 instances are associated with each combination of numbers of items in the set $\{10,20,30,50\}$ and bins in the set $\{(250,250),(500,500),(1000,1000)\}$. The dimensions of the items were generated following a uniform distribution. The last instance corresponds to a real-world problem with 32 items and bin $(3000,3000)$. In all instances, profits coincide with item areas;

- OF: two small instances generated by Oliveira and Ferreira [39], where item dimensions follow a beta distribution and profits coincide with item areas;

- OKP: five instances generated by Fekete and Schepers [21] in the same manner as the NGCUT set. The number of items ranges between 15 and 33 and the width and height of the bins is 100 ;

- CU and CW: 22 instances generated by Fayard et al. [20]. The number of items ranges between 25 and 60 and the largest bin is $(992,970)$. CU has 11 unweighted instances (i.e., with item profits given by their area), whereas CW has 11 weighted instances, where profits are randomly generated;

- LU, LW, LX: three groups of 5 large-scale instances each, proposed by Hifi [23] and Russo et al. [42]. The number of items ranges between 100 and 550 and the largest bin is (45 237, 35983$)$;

- APT: 40 instances proposed by Alvarez-Valdés et al. [2]. The range of number of items and dimensions of the bin follows the policies adopted for $\mathrm{CU}$ and $\mathrm{CW}$, but the instances also contain some smaller items to increase their difficulty;

- NGCUTFS: 630 instances generated by Beasley [7] in the same manner as the OKP set, but with the number of items ranging between 40 and 1000;

- MP: 450 instances proposed by Morabito and Pureza [37]. The number of items is in $\{10,20,30,40,50\}$ and the width and height of the bins is 100 . This set is divided into three classes of 150 instances and each class is characterized by different ranges used to create the item dimensions;

- VU: 80 instances proposed by Velasco and Uchoa [46]. The number of items is 50, and the bins have dimensions ranging between 100 and 400 . The items are relatively small compared to the bin, a feature often associated with hard instances.

\subsection{Benchmarks proposed for the 2D-OPP}

- CJCM: 42 instances proposed by Clautiaux et al. [16]. The number of items ranges between 10 and 23 and the width and height of the bins is 20 ;

- MSB: 1080 instances proposed by Mesyagutov et al. [36], divided into two classes. The first class has 630 instances, each with 20 items and width and height of the bins equal to 1000 . The second has 450 instances, each with number of items in $\{10,15,20,25,30\}$ and width and height of the bins equal to 100 . 


\section{Bibliographies and additional tools}

The 2DPackLib includes

- TwoBinPack, an open source software to interactively solve rectangular cutting and packing problems, developed by Costa et al. [17], an application useful to practitioners and developers thanks to its visual tools;

- the BibTeX file containing almost 200 references, mostly appeared in the last ten years, from the survey by Iori et al. [26];

- a list of links related to two-dimensional cutting and packing, such as working groups, library pages, and the instance generator developed by Silva et al. [43].

\section{Conclusions}

We presented the 2DPackLib, a library dedicated to two-dimensional orthogonal cutting and packing problems that provides pointers to surveys and typologies, benchmarks, an interactive visual tool, a BibTeX file of almost 200 references, and a list of relevant links. The benchmarks (comprising over 3000 instances) are standardized to provide a unified format for all the instances. This addresses previous issues on benchmarks with different formats, not always clearly detailed.

We are confident that the 2DPackLib will facilitate future research in the active area of two-dimensional cutting and packing problems.

Acknowledgements This research was supported by the Air Force Office of Scientific Research under Grant no. FA8655-20-1-7012 and by the São Paulo Research Foundation under Grant no. 2017/11831-1.

Funding Open access funding provided by Alma Mater Studiorum - Università di Bologna within the CRUI-CARE Agreement.

\section{Declarations}

Conflict of interest The authors declare that they have no conflict of interest.

Data availability The datasets generated during and/or analyzed during the current study are available in the 2DPackLib repository, http://or.dei.unibo.it/library/2dpacklib.

Open Access This article is licensed under a Creative Commons Attribution 4.0 International License, which permits use, sharing, adaptation, distribution and reproduction in any medium or format, as long as you give appropriate credit to the original author(s) and the source, provide a link to the Creative Commons licence, and indicate if changes were made. The images or other third party material in this article are included in the article's Creative Commons licence, unless indicated otherwise in a credit line to the material. If material is not included in the article's Creative Commons licence and your intended use is not permitted by statutory regulation or exceeds the permitted use, you will need to obtain permission directly from the copyright holder. To view a copy of this licence, visit http://creativecommons.org/licenses/by/4.0/.

\section{References}

1. Alvarez-Valdes, R., Carravilla, M., Oliveira, J.: Cutting and packing. In: Martí, R., Pardalos, P., Resende, M. (eds.) Handbook of Heuristics, pp. 931-977. Springer (2018) 
2. Alvarez-Valdés, R., Parajón, A., Tamarit, J.: A tabu search algorithm for large-scale guillotine (un)constrained two-dimensional cutting problems. Comput. Oper. Res. 29(7), 925-947 (2002)

3. Applegate, D., Bixby, R., Chvatal, V., Cook, W.: The Traveling Salesman Problem-A Computational Study. Princeton University Press, Princeton (2006)

4. Beasley, J.: Algorithms for unconstrained two-dimensional guillotine cutting. J. Oper. Res. Soc. 36(4), 297-306 (1985)

5. Beasley, J.: An exact two-dimensional non-guillotine cutting tree search procedure. Oper. Res. 33(1), 49-64 (1985)

6. Beasley, J.: OR-Library: distributing test problems by electronic mail. J. Oper. Res. Soc. 41(11), 10691072 (1990)

7. Beasley, J.: A population heuristic for constrained two-dimensional non-guillotine cutting. Eur. J. Oper. Res.D 156(3), 601-627 (2004)

8. Bengtsson, B.E.: Packing rectangular pieces-a heuristic approach. Comput. J. 25, 353-357 (1982)

9. Berkey, J.O., Wang, P.Y.: Two dimensional finite bin packing algorithms. J. Oper. Res. Soc. 38, 423-429 (1987)

10. Bezerra, V., Leao, A., Oliveira, J., Santos, M.: Models for the two-dimensional level strip packing problem-a review and a computational evaluation. J. Oper. Res. Soc. 71(4), 606-627 (2020)

11. Burkard, R., Karisch, S., Rendl, F.: QAPLIB- a quadratic assignment problem library. Eur. J. Oper. Res. 55(1), 115-119 (1991)

12. Burkard, R., Karisch, S., Rendl, F.: QAPLIB - a quadratic assignment problem library. J. Global Optim. 10, 391-403 (1997)

13. Burke, E., Kendall, G., Whitwell, G.: A new placement heuristic for the orthogonal stock-cutting problem. Oper. Res. 52(4), 655-671 (2004)

14. Christensen, H.I., Khan, A., Pokutta, S., Tetali, P.: Approximation and online algorithms for multidimensional bin packing: a survey. Comput. Sci. Rev. 24, 63-79 (2017)

15. Christofides, N., Whitlock, C.: An algorithm for two-dimensional cutting problems. Oper. Res. 25(1), 30-44 (1977)

16. Clautiaux, F., Jouglet, A., Carlier, J., Moukrim, A.: A new constraint programming approach for the orthogonal packing problem. Comput. Oper. Res. 35(3), 944-959 (2008)

17. Costa, G., Delorme, M., Iori, M., Malaguti, E., Martello, S.: Training software for orthogonal packing problems. Comput. Ind. Eng. 111, 139-147 (2017)

18. Delorme, M., Iori, M., Martello, S.: BPPLIB: a library for bin packing and cutting stock problems. Optim. Lett. 12(2), 235-250 (2018)

19. Dyckhoff, H.: A typology of cutting and packing problems. Eur. J. Oper. Res. 44(2), 145-159 (1990)

20. Fayard, D., Hifi, M., Zissimopoulos, V.: An efficient approach for large-scale two-dimensional guillotine cutting stock problems. J. Oper. Res. Soc. 49(12), 1270-1277 (1998)

21. Fekete, S., Schepers, J.: A new exact algorithm for general orthogonal d-dimensional knapsack problems. In: Burkard, R., Woeginger, G. (eds.) Algorithms - ESA, vol. 1284, pp. 144-156. Springer (1997)

22. Groër, C., Golden, B., Wasil, E.: A library of local search heuristics for the vehicle routing problem. Math. Programm. Comput. 2, 79-101 (2010)

23. Hifi, M.: Exact algorithms for large-scale unconstrained two and three staged cutting problems. Combinatorial Optim. Appl. 18, 63-88 (2001)

24. Hopper, E., Turton, B.: An empirical investigation of meta-heuristic and heuristic algorithms for a 2D packing problem. Eur. J. Oper. Res. 128(1), 34-57 (2001)

25. Hopper, E., Turton, B.: Problem generators for rectangular packing problems. Stud. Inform. Univ. 2, 123-136 (2002)

26. Iori, M., de Lima, V., Martello, S., Miyazawa, F., Monaci, M.: Exact solution techniques for twodimensional cutting and packing. Eur. J. Oper. Res. 289(2), 399-415 (2021)

27. Koch, T., Achterberg, T., Andersen, E., Bastert, O., Berthold, T., Bixby, R., Danna, E., Gamrath, G., Gleixner, A., Heinz, S., Lodi, A., Mittelmann, H., Ralphs, T., Salvagnin, D., Steffy, D., Wolter, K.: MIPLIB 2010. Math. Program. Comput. 3, 103 (2011)

28. Leung, S., Zhang, D.: A fast layer-based heuristic for non-guillotine strip packing. Expert Syst. Appl. 38(10), 13032-13042 (2011)

29. Lodi, A., Martello, S., Monaci, M.: Two-dimensional packing problems: a survey. Eur. J. Oper. Res. 141(2), 241-252 (2002) 
30. Lodi, A., Martello, S., Monaci, M., Vigo, D.: Two-dimensional bin packing problems. In: Paschos, V.T. (ed.) Paradigms of Combinatorial Optimization: Problems and New Approaches, pp. 107-129. Wiley, Hoboken (2014)

31. Lodi, A., Martello, S., Vigo, D.: Heuristic and metaheuristic approaches for a class of two-dimensional bin packing problems. INFORMS J. Comput. 11(4), 345-357 (1999)

32. Lodi, A., Martello, S., Vigo, D.: Recent advances on two-dimensional bin packing problems. Discrete Appl. Math. 123(1), 379-396 (2002)

33. Macedo, R., Alves, C., Valério de Carvalho, J.: Arc-flow model for the two-dimensional guillotine cutting stock problem. Comput. Oper. Res. 37(6), 991-1001 (2010)

34. Martello, S., Monaci, M., Vigo, D.: An exact approach to the strip-packing problem. INFORMS J. Comput. 15(3), 310-319 (2003)

35. Martello, S., Vigo, D.: Exact solution of the two-dimensional finite bin packing problem. Manag. Sci. 44(3), 388-399 (1998)

36. Mesyagutov, M., Scheithauer, G., Belov, G.: LP bounds in various constraint programming approaches for orthogonal packing. Comput. Oper. Res. 39(10), 2425-2438 (2012)

37. Morabito, R., Pureza, V.: A heuristic approach based on dynamic programming and and/or-graph search for the constrained two-dimensional guillotine cutting problem. Ann. Oper. Res. 179, 297-315 (2010)

38. Ntene, N., van Vuuren, J.: A survey and comparison of guillotine heuristics for the $2 \mathrm{D}$ oriented offline strip packing problem. Discrete Optim. 6(2), 174-188 (2009)

39. Oliveira, J., Ferreira, J.: An improved version of Wangs algorithm for two-dimensional cutting problems. Eur. J. Oper. Res. 44(2), 256-266 (1990)

40. Oliveira, J., Júnior, A.N., Silva, E., Carravilla, M.: A survey on heuristics for the two-dimensional rectangular strip packing problem. Pesquisa Operacional 36(2), 197-226 (2016)

41. Russo, M., Boccia, M., Sforza, A., Sterle, C.: Constrained two-dimensional guillotine cutting problem: upper-bound review and categorization. Int. Trans. Oper. Res. 27(2), 794-834 (2020)

42. Russo, M., Sforza, A., Sterle, C.: An exact dynamic programming algorithm for large-scale unconstrained two-dimensional guillotine cutting problems. Comput. Oper. Res. 50, 97-114 (2014)

43. Silva, E., Oliveira, J., Wäscher, G.: 2DCPackGen: A problem generator for two-dimensional rectangular cutting and packing problems. Eur. J. Oper. Res. 237(3), 846-856 (2014)

44. Silva, E., Oliveira, J., Wäscher, G.: The pallet loading problem: a review of solution methods and computational experiments. Int. Trans. Oper. Res. 23(1-2), 147-172 (2016)

45. Uchoa, E., Pecin, D., Pessoa, A., Poggi, M., Vidal, T., Subramanian, A.: New benchmark instances for the capacitated vehicle routing problem. Eur. J. Oper. Res. 257(3), 845-858 (2017)

46. Velasco, A., Uchoa, E.: Improved state space relaxation for constrained two-dimensional guillotine cutting problems. Eur. J. Oper. Res. 272(1), 106-120 (2019)

47. Wang, P.: Two algorithms for constrained two-dimensional cutting stock problems. Oper. Res. 31(3), 573-586 (1983)

48. Wäscher, G., Haußner, H., Schumann, H.: An improved typology of cutting and packing problems. Eur. J. Oper. Res. 183(3), 1109-1130 (2007)

Publisher's Note Springer Nature remains neutral with regard to jurisdictional claims in published maps and institutional affiliations. 